\title{
BMJ Open Early prediction of physical activity level 1 year after stroke: a longitudinal cohort study
}

\author{
Ola A Olsson, Hanna C Persson, Margit Alt Murphy, Katharina S Sunnerhagen
}

To cite: Olsson $O A$, Persson HC, Alt Murphy M, et al. Early prediction of physical activity level 1 year after stroke: a longitudinal cohort study. BMJ Open 2017;7:e016369. doi:10.1136/ bmjopen-2017-016369

- Prepublication history and additional material for this paper are available online. To view these files please visit the journal online (http://dx.doi. org/10.1136/bmjopen-2017016369).

Received 9 February 2017 Revised 12 June 2017 Accepted 21 June 2017

CrossMark

Department of Clinical Neuroscience, Institute of Neuroscience and Physiology, Sahlgrenska Academy at University of Gothenburg, Gothenburg, Sweden

Correspondence to

Prof. Katharina S Sunnerhagen; ks.sunnerhagen@neuro.gu.se

\section{ABSTRACT}

Objective To investigate which variables present prior and early after stroke may have an impact on the level of physical activity $(\mathrm{PA}) 1$ year poststroke.

Design Prospective longitudinal cohort and logistic regression analysis.

Setting Stroke Unit at Sahlgrenska University Hospital, Gothenburg, Sweden.

Participants 117 individuals as part of the Stroke Arm Longitudinal Study (SALGOT) admitted to the stroke unit during a period of 18 months were consecutively recruited. The inclusion criteria were: first-time stroke, impaired upper extremity function, admitted to the stroke unit within 3 days since onset, local residency and $\geq 18$ years old. The exclusion criteria were: upper extremity condition or severe multi-impairment prior to stroke, short life expectancy and non-Swedish speaking. 77 participants followed up at 1 year poststroke were included in the analysis.

Primary outcome PA level 1 year after stroke was assessed using a 6-level Saltin-Grimby Scale, which was first dichotomised into mostly inactive or mostly active and second into low or moderate/high level of PA.

Results Being mostly inactive 1 year after stroke could be predicted by age at stroke onset $(\mathrm{OR} 1.07,95 \% \mathrm{Cl} 1.00$ to $1.13, p=0.041)$, functional dependency at discharge (OR $7.01,95 \% \mathrm{Cl} 1.73$ to $28.43, \mathrm{p}=0.006$ ) and prestroke PA (OR $7.46,95 \% \mathrm{Cl} 1.51$ to $36.82, p=0.014$ ). Having a low level of PA 1 year after stroke could be predicted by age at stroke onset (OR 1.13, 95\% Cl 1.06 to $1.21, \mathrm{p}<0.001)$ and functional dependency at discharge $(0 \mathrm{R} 3.62,95 \% \mathrm{Cl} 1.09$ to $12.04, p=0.036$ ).

Conclusions Previous low level of PA, older age and functional dependency all provided value in predicting Iow PA 1 year after stroke. These results indicate that age and simple clinical evaluations early after stroke may be useful to help clinicians identify persons at risk of being insufficiently active after stroke. Further research is needed to clarify if these findings may apply to the large population of stroke survivors.

Trial registration number ClinicalTrials.gov (NCT01115348).

\section{INTRODUCTION}

Low physical activity (PA) has shown to be an independent risk factor for stroke, ${ }^{1-3}$ and PA is a part of primary ${ }^{1}$ as well as secondary prevention in most of the stroke guidelines. ${ }^{4}$ The WHO has identified physical

\section{Strengths and limitations of this study}

- Clinically important parameters prior to and early after stroke were included.

- Longitudinal consecutively recruited cohort study with 1-year follow-up time.

- Clinically relevant dichotomisation of physical activity levels produced interpretable data.

- Despite relatively large cohort, the number of included predictors was limited due to small number of cases for some variables.

- Persons with minor stroke showing no upper extremity impairment early after stroke were not included.

inactivity to be the fourth leading risk factor for overall global mortality. ${ }^{5}$ The definition of PA according to WHO is 'any bodily movement produced by skeletal muscles that requires energy expenditure including activities undertaken while working, playing, carrying out household chores, travelling and engaging in recreational pursuits'. ${ }^{6}$ Higher PA level prestroke may predict a less severe stroke ${ }^{78}$ decrease the overall risk for death from first time stroke ${ }^{9}$ and is associated with a better functional status poststroke. 71011

It is a complex question to answer why some people are physically active after having a stroke and others are not. PA in healthy populations has shown to be influenced by factors such as age, gender, motivation, previous PA, self-efficacy and health status. ${ }^{12}$ Being physically active poststroke is associated with a better quality of life and has a positive correlation to functional ability. ${ }^{14}$ The PA level among stroke survivors has been shown to be significantly lower than in a healthy reference population ${ }^{15-19}$ and correlates with walking ability, balance and physical fitness, ${ }^{15}$ but cannot be explained by motor disability alone. ${ }^{1620}$ Barriers to PA reported by stroke survivors include lack of motivation, fear of falling, inaccessibility to training 
centres and physical impairments. ${ }^{21}{ }^{22}$ It is, however, not clear to what extent factors connected to the prestroke lifestyle and medical status may be associated with the PA level among stroke survivors. Identifying persons at risk of being inadequately active poststroke may help to target specific interventions for this group at an early stage. The purpose of this study was to investigate which possible prestroke and early predictor variables may impact the level of PA 1 year after the first-time stroke.

\section{MATERIALS AND METHODS}

\section{Population and data collection}

This longitudinal study is a part of the Stroke Arm Longitudinal (SALGOT) Study at the University of Gothenburg, ${ }^{23}$ with the original purpose to describe upper extremity functioning after stroke. Over a period of 18 months, in 2009-2010, consecutively, every person who met the criteria was included to the SALGOT Study from one of the largest out of three comprehensive Stroke Units at the Sahlgrenska University Hospital, Gothenburg. The following inclusion criteria were used: (1) first-time stroke according to International Classification of Diseases codes I61 intracerebral haemorrhage or I63 ischaemic stroke; (2) impaired upper extremity function, defined as not achieving the maximal points at the Action Research Arm Test (ARAT) ${ }^{24} 3$ days poststroke; (3) admitted to the Stroke unit within 3 days since stroke onset; (4) residency in the Gothenburg urban area, within $35 \mathrm{~km}$ from the hospital and (5) $\geq 18$ years of age. The exclusion criteria were: (1) an upper extremity injury/condition prior to stroke; (2) severe multi-impairments or diminished physical condition prior to stroke; (3) short life expectancy and (4) non-Swedish speaking. Three experienced physiotherapists performed all clinical assessments according to a standardised protocol. ${ }^{23}$

In SALGOT, the patients were assessed at admission and discharge as well as at 3 and 10 days; at 3, 4 and 6 weeks; and at 3, 6 and 12 months poststroke. In the current study, data from admission, discharge, 3 days and 12 months were used. Most assessments were performed at the hospital and only at persons' home or nursing home when the participant was unable to travel. Prior power analysis for SALGOT to determine a minimum of 6 points change on ARAT (statistical power of $0.8, \alpha=0.05$ ) and considering a $30 \%$ dropout rate indicated that a sample size of 114 was needed. From a total cohort of 763 persons, 117 were included in the SALGOT study and 77 still remained in the study at 1 year poststroke (figure 1). The main reason for not being assessed at 1 year was death $(\mathrm{n}=14)$ (figure 1$)$. The study was approved by the Regional Ethical Review Board in Gothenburg (225-08). All participants or their next of kin gave written informed consent. The STROBE (Strengthening The Reporting of OBservational Studies in Epidemiology) guidelines for reporting observational data were followed. ${ }^{25}$

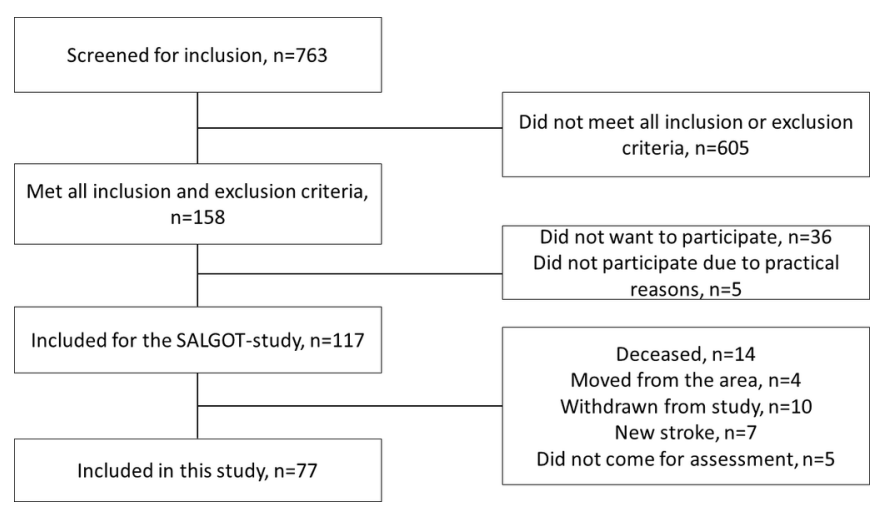

Figure 1 Flowchart for inclusion of the study participants.

\section{Potential predictor variables}

Potential predictors prior and close to the stroke onset, theorised to have impact on PA, were considered for model building. ${ }^{13} 15$ Prior stroke predictor variables included in the analyses were: smoking, living alone, transient ischaemic attack (TIA), diabetes, atrial fibrillation, treatment for high blood pressure and PA level. Other predictors included were: age, gender, type of stroke, stroke severity, upper extremity functioning 3 days poststroke and functional dependency at discharge (Modified Rankin Scale (mRS)), shown in table 1.

Information of history of smoking, whether the participant shared livings with another adult and medical history prior to stroke were acquired by the national Swedish Stroke Register ${ }^{26}$ or medical charts. The stroke severity at admission to the hospital was assessed using the National Institute of Health Stroke Scale (NIHSS). ${ }^{27}$ The upper extremity functioning was assessed using the ARAT, which includes 19 items scored on a four-grade ordinal scale, with a total score varying from 0 to 57 points, where a higher score indicates less limitation. ${ }^{24}$ The functional dependency at discharge from the stroke unit (mean time 13 days, SD 7,4 range 1-42) was assessed using the mRS. ${ }^{28}$ The $\mathrm{mRS}$ is an ordinal scale ranging from 0 to 6 where lower numbers indicate less dependency. ${ }^{28}$ The mRS was dichotomised between the grade 3 and 4 creating one group that contained persons able to walk without assistance (no/slight/moderate disability, grades 1-3) and one group who could not (moderately severe to severe disability, grades $4-5$ ). The self-reported PA level was recorded using a 6-level scale for classification of PA level (including leisure time, occupational and household activities) (online supplementary appendix A), originally developed from the four- graded Saltin-Grimby Scale. ${ }^{29} 30$ The participants' PA level was scored through an interview within 3 days and at 1 year poststroke considering the PA level during the previous 6 months. In the statistical analyses, the PA was dichotomised in two different ways. First, to mostly inactive (grade 1-2) or mostly active (grade 3-5) and; second, to low (grade 1-3) or moderate/high activity level (4-5). The first dichotomisation was selected to match the original four-level scale based on prevention of cardiovascular disease. ${ }^{31}$ The second dichotomisation 


\begin{tabular}{|c|c|}
\hline \multicolumn{2}{|l|}{$\begin{array}{l}\text { Demographic and clinical characteristics } \\
(\mathrm{N}=77)\end{array}$} \\
\hline Age at stroke onset, years, mean (SD) & $67.2(11.9)$ \\
\hline Men, n (\%) & $46(59.7)$ \\
\hline Haemorrhagic stroke, ${ }^{\star} \mathrm{n}(\%)$ & $11(14.3)$ \\
\hline Smoking, ${ }^{*} \dagger \mathrm{n}(\%), \mathrm{n}=76$ & $18(23.7)$ \\
\hline Living alone, ${ }^{*} \mathrm{n}(\%)$ & $31(40.3)$ \\
\hline TIA/amaurosis fugax, ${ }^{*} \dagger \mathrm{n}(\%), \mathrm{n}=76$ & $4(5.3)$ \\
\hline Diabetes, ${ }^{*} † \mathrm{n}(\%)$ & $10(13)$ \\
\hline Atrial fibrillation, ${ }^{\star} \dagger \mathrm{n}(\%), \mathrm{n}=76$ & $11(14.5)$ \\
\hline $\begin{array}{l}\text { Treatment for high blood pressure, }{ }^{*} n(\%) \text {, } \\
n=76\end{array}$ & $26(34.2)$ \\
\hline NIHSS at admission, median (q1-q3) & $7(3-12.5)$ \\
\hline ARAT at 3 days, median ( $q 1-q 3$ ), $n=74$ & $7(0-47)$ \\
\hline \multicolumn{2}{|l|}{$\mathrm{mRS}$ at discharge from stroke unit, $\mathrm{n}(\%)$} \\
\hline Independent walkers (grade 0-3) & $37(48.1)$ \\
\hline Unable to walk independently (grade $4-5$ ) & $40(51.9)$ \\
\hline \multicolumn{2}{|l|}{ Prestroke PA, $n(\%), n=73$} \\
\hline Mostly inactive (grade 1-2) & $19(26.0)$ \\
\hline Low (grade 1-3) & $43(58.9)$ \\
\hline Acute hospital stay, days, mean (SD) & $12.6(7.1)$ \\
\hline \multicolumn{2}{|l|}{$\begin{array}{l}\text { Discharge to postacute hospital stay, days, } n \\
(\%)\end{array}$} \\
\hline Ordinary home & $27(35)$ \\
\hline In-hospital rehabilitation unit & $46(60)$ \\
\hline Nursing home & $4(5)$ \\
\hline
\end{tabular}

*Prior to stroke, $†$ Not included in the prediction models due to too few observations.

ARAT, Action Research Arm Test; mRS, Modified Rankin Scale; NIHSS, National Institute of Stroke Scale; PA, physical activity; q1q3, first to third quartile; TIA, transient ischaemic attack; $y / n, y e s /$ no.

was selected to match the level of PA (of 30 min of activity, 5 days per week) recommended by the WHO in order to prevent morbidity. ${ }^{6}$ Within each prediction model, the same dichotomisation of PA level was used for outcome and for predictor variable.

\section{Statistics}

Differences between groups were investigated with Fisher's exact test, Mann-Whitney U test or t-test depending on data level. Demographic data were presented with medians and percentiles or means and SD. The statistically significant level was set to $\mathrm{p}<0.05$ unless stated otherwise. A multivariate logistic regression was used to investigate which predictor variables may impact on the PA level 1 year after stroke. Two separate models were built, one for each dichotomisation of the outcome variable. As first step in selection of potential predictor variables for the regression models, the cross tabulation was used to identify and exclude predictor variables with less than five observations in any subgroup. Collinearity between predictor variables was checked for using Spearman's rank correlation test for ordinal variables or likelihood ratio test (LRT) for binary variables. Variables with correlation above 0.7 were considered for collinearity. Second step was a series of univariate logistic regression analysis performed on all variables not excluded by the cross tabulation in order to identify significant variables for further analyses (significance level $p<0.25$, tested with Wald's test). Third, the variables that were significant in the univariate step was put in multivariate models, built on the enter method in which all predictor variables not reaching the significance level of 0.05 were ruled out. Individuals with missing data on any of the variables included in the final multivariate models were excluded for analysis (table 1). Fourth, all of the previously ruled out variables were then reinserted in the final model one by one to check for possible significant effect in the model $(\mathrm{p}<0.05, \mathrm{LRT})$. Finally, the models were analysed with the LRT, percent of correct classification, Nagelkerke $\mathrm{R}^{2}$ and the Hosmer and Lemeshow goodness of fit test. Results are presented as OR with 95\% CI. Data were analysed using the Statistical Package for Social Sciences (SPSS) software.

\section{RESULTS \\ Clinical characteristics}

The group of non-participants not assessed at 1 year from the SALGOT cohort $(n=40)$ was older (mean difference 6.23 years, $\mathrm{p}=0.01$ ), had a higher incidence of atrial fibrillation $(\mathrm{p}=0.04)$ and were less active prior to their stroke $(p=0.03)$. No other statistical significant differences were found between the groups. Demographic and clinical characteristics are presented in table 1. Prior to stroke, $74 \%(\mathrm{n}=54)$ of the participants were considered to be mostly active, in contrast to $61 \%(\mathrm{n}=47)$ at 1 year poststroke. Similarly, $41 \%(\mathrm{n}=30)$ of the participants had a moderate to high activity level prior to stroke in contrast to $34 \%(n=26) 1$ year later.

\section{Selection of predictor variables}

The type of stroke along with smoking, TIA, diabetes and atrial fibrillation prior to stroke contained too few individuals in subgroups and were, therefore, not included into further analysis. Strong significant collinearity was found between the predictor variables: mRS and ARAT $(-0.74)$. These two variables were, therefore, entered into separate models and their impact to respective model compared. Thus, seven possible predictor variables were considered to be entered in the multivariate models in this second step. LRT showed a significant correlation between gender and prestroke PA (LRT $=5.910, \mathrm{p}=0.02$ and between treatment for high blood pressure prior to stroke and prestroke PA (LRT=10.358, $\mathrm{p}=0.01)$. The results from the univariate analysis are presented in an online supplementary appendix B. None of the variables that were reinserted 
Table 2 Logistic regression models for predicting PA level 1 year poststroke

$\begin{array}{lllllll}\text { Coefficient } & \text { B } & \text { SE } & \text { Wald's test } & \text { df } & p & \text { OR }(95 \% \mathrm{Cl})\end{array}$

\begin{tabular}{|c|c|c|c|c|c|c|}
\hline \multicolumn{7}{|c|}{ (a) Dependent variable of mostly inactive $(n=73)$} \\
\hline Constant & -6.52 & 2.15 & 9.17 & 1 & 0.002 & 0.001 \\
\hline Age & 0.06 & 0.03 & 4.18 & 1 & 0.041 & 1.07 (1.00 to 1.13$)$ \\
\hline $\mathrm{mRS}$ at discharge & 1.95 & 0.71 & 7.43 & 1 & 0.006 & 7.01 (1.73 to 28.43 ) \\
\hline $\begin{array}{l}\text { Prestroke PA (mostly } \\
\text { inactive) }\end{array}$ & 2.01 & 0.81 & 6.10 & 1 & 0.014 & 7.46 (1.51 to 36.82$)$ \\
\hline Test & & & $\mathrm{X}^{2}$ & df & $\mathrm{p}$ & \\
\hline Likelihood ratio test & & & 32.59 & 3 & $<0.001$ & \\
\hline Hosmer and Lemeshon & & & 9.66 & 8 & 0.290 & \\
\hline \multicolumn{7}{|c|}{ (b) Dependent variable of low level of PA ( $n=77$ ) } \\
\hline Constant & -8.12 & 2.25 & 13.03 & 1 & $<0.001$ & $<0.001$ \\
\hline Age & 0.13 & 0.03 & 13.52 & 1 & $<0.001$ & $1.13(1.06$ to 1.21$)$ \\
\hline $\mathrm{mRS}$ at discharge & 1.29 & 0.61 & 4.41 & 1 & 0.036 & 3.62 (1.09 to 12.04$)$ \\
\hline Test & & & $x^{2}$ & df & $\mathrm{p}$ & \\
\hline Likelihood ratio test & & & 30.47 & 2 & $<0.001$ & \\
\hline Hosmer and Lemeshon & & & 3.28 & 7 & 0.858 & \\
\hline
\end{tabular}

Dependent variable coded as (a) mostly active $=0$, mostly inactive $=1$; (b) moderate/high $P A=0$, low $P A=1$; Cox and $S$ nell $R^{2}(a)=0.360$; $(b)=0.327$ Nagelkerke $R^{2}(a)=0.489$; $(b)=0.453$.

PA, physical activity; mRS, Modified Rankin Scale.

in the final step for the multivariate analysis were significant $(\mathrm{p}>0.05)$.

\section{Predicting being mostly inactive}

The final model for predicting being mostly inactive poststroke included three significant predictor variables: age, functional dependency (mRS) and prestroke PA (table 2a).

The percentage of total correctly classified for the model was 78.1 with sensitivity $75.0 \%$ and specificity of $79.5 \%$. The odds for being mostly inactive 1 year after stroke, increased by $7 \%$ for every year of increasing age. The odds for being inactive also increased by 6 times if the participant was not able to walk independently at discharge and by 6.5 times if the participant was already mostly inactive prestroke. Predicted probabilities for this model are presented in figure 2 . As seen in figure 2 , there were no observations on mostly inactive non-walkers below age 70 years, which means that the predicted probabilities are extrapolated below this age. A separate model including the three significant predictor variables, age, ARAT (instead of mRS) and prestroke PA demonstrated comparable level of correct classification (78.6\%).

\section{Predicting low PA}

The final model for predicting low PA level included two significant predictor variables: age and functional dependency (mRS) at discharge from stroke unit (table $2 b)$.

The percentage of total correctly classified for the model was 74.0 with sensitivity $77.2 \%$ and specificity of $65.0 \%$. The odds of having a low PA level 1 year after stroke increased with $13 \%$ for every year of increasing age. The odds of having a low PA level also increased, by 2.6 times if the participant was not able to walk independently at discharge. Predicted probabilities for this model are presented in figure 3 . A separate model including the two significant predictor variables, age and ARAT (instead of mRS) demonstrated comparable level of correct classification $(75.7 \%)$.

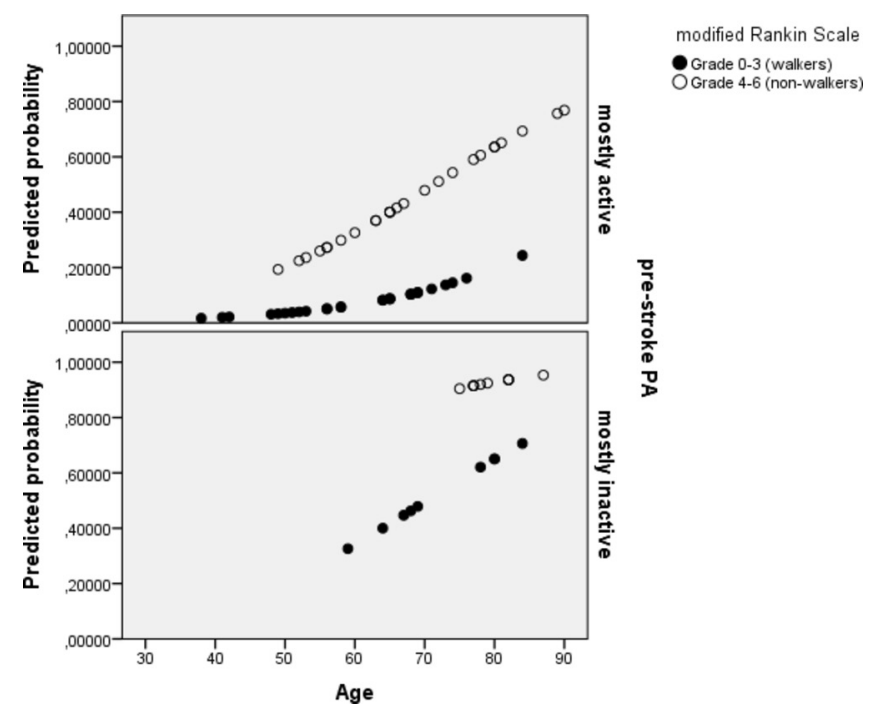

Figure 2 Predicted probabilities of being mostly inactive 1 year after stroke. The predicted probability increases with higher age, higher degree of functional dependency and being physically inactive prestroke. 


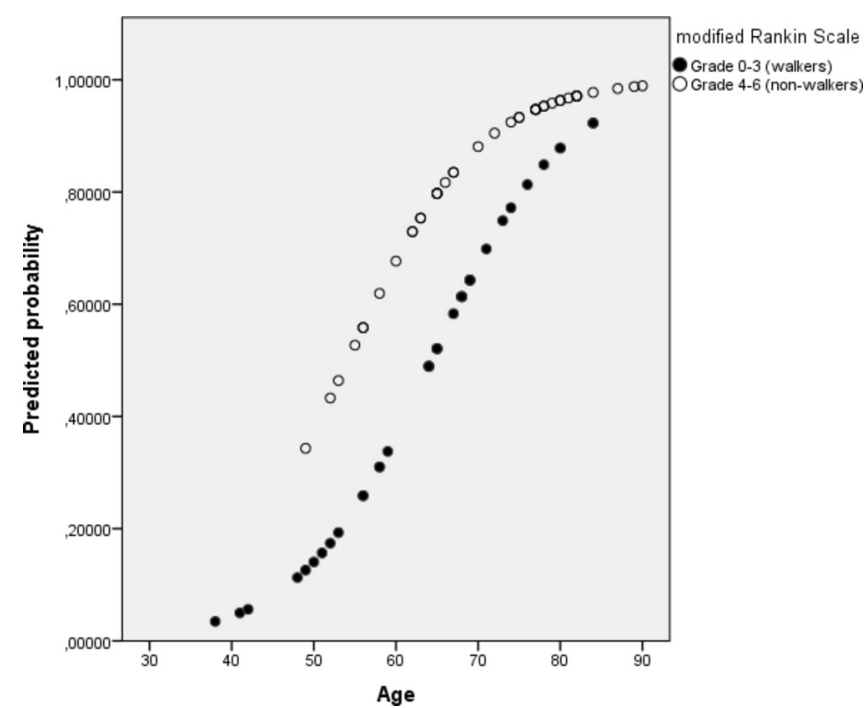

Figure 3 Predicted probability for having low PA 1 year after stroke. The predicted probability increases with higher age and higher degree of functional dependency.

\section{DISCUSSION}

Higher age, functional dependency at discharge from stroke unit and being physically inactive prior to stroke all contributed to increase the probability of being physically inactive 1 year after stroke. The probability of having a low PA level after stroke increased with older age and functional dependency at discharge from stroke unit. Findings from this study provide new insights on what factors obtained early after stroke may impact on the PA level at later stages among stroke survivors. This knowledge could be used to identify patients at risk for inactivity or low PA level early after stroke, so that targeted intervention could be offered as part of secondary prevention.

When comparing levels of PA, two different dichotomisations of data (two models) based on different recommendations on PA was used. ${ }^{629}$ The first model aimed to address inactivity as important cut-off for prevention of cardiovascular disease ${ }^{31}$ and the second to address $\mathrm{PA}$ at lower than the recommended level required for prevention of morbidity. ${ }^{6}$ Age was found to be a significant predictor in both models, but it had a greater impact on the model for identifying those with low PA level. This finding is in concurrence with an earlier study in older adults, where the age was inversely correlated with the amount of moderate-intensity PA, but not with the amount of low-intensity PA. ${ }^{32}$ Functional dependency, including ability to walk independently or not, was also found as a significant predictor for PA after stroke in both models, which is in concurrence with previous studies. ${ }^{1521}$ These findings suggest that, similarly to older adults, age may have an impact on the intensity of PA after stroke but also that the disability level expressed as dependence in walking and daily activities influence the PA level at later stages poststroke. The upper extremity functioning (ARAT) early after stroke was found to have similar effect on the later poststroke PA, as the functional dependency $(\mathrm{mRS})$ at discharge. Functional dependency at discharge and limitation in the upper extremity use early after stroke may both be associated with the stroke severity, but these factors may also mean that the limited function itself after stroke may impact the PA level negatively. ${ }^{15-19}$ Being mostly inactive prestroke had a significant effect when predicting inactivity at later stage poststroke. However, the level of PA prestroke, low or moderate/high, did not have a significant effect in the model predicting poststroke PA level, which indicates that the level of PA poststroke may to larger degree be affected by other factors, such as the disability level, age and comorbidities.

There has been little interest in investigating which early predictors might influence PA among stroke survivors and most studies on PA look at cross-sectional correlations. A previous longitudinal study, ${ }^{33}$ investigating physical inactivity after stroke, found significant correlation between time spent upright and degree of independence in activities of daily living and walking at the first weeks after stroke, as well as at 1, 2 and 3 years poststroke. Although these findings reflect merely cross-sectional correlations, they indicate that independence in daily activities and ambulation are important for PA among stroke survivors. In a review comprising people after stroke with ability to walk, ${ }^{15}$ walking ability, balance and physical fitness were positively associated with PA level. Walking ability in the form of walking speed has further been found to explain some of the variation of PA level among stroke survivors. ${ }^{16}$ Studies on what stroke survivors experience as barriers to PA have identified physical impairment as one of the main barriers to $\mathrm{PA},{ }^{21} 22$ yet motor impairment has been found to correlate mainly with walking capacity and energy cost for walking and not with PA level. ${ }^{17}$ In another study physical capacity, measured by a test for fitness, was found to have a moderate correlation to self-assessed PA. ${ }^{34}$ In our study, the mRS addressing disability rather than impairment was used ${ }^{28}$ and although functional disability and motor impairment are correlated, impairment does not fully explain disability among people with stroke. ${ }^{20}$ Previous studies have not shown significant correlation between age and PA after stroke. ${ }^{15} 33$ Age has, however, been found to be inversely correlated to PA in healthy populations, ${ }^{12}{ }^{35}$ although not as a clear determinant compared with health status or previous PA habits. ${ }^{12}$ The decline in PA with increasing age does not seem to be linear but exponential in older adults ${ }^{35}$ and functional outcome has been found to drop steeply in the older ages among people who has had stroke, ${ }^{36}$ yet most work on PA among stroke survivors have been made in persons aged $65-75$ years. ${ }^{15}$ The present study had no upper limit of age, yet the participants in the study were somewhat younger than the average stroke population in Sweden; ${ }^{26}$ therefore, the effect of age on PA level in stroke survivors might be slightly underestimated.

Prestroke PA has been found to have a significant impact on functional outcome at acute phase, ${ }^{11} 3$ months ${ }^{10} 19$ 1 year $^{11}$ and 2 years after stroke. ${ }^{7}$ A longitudinal study ${ }^{11}$ showed that the main differences for functional outcome were found when comparing a subgroup with relatively 
low PA level, measured as people who walked less than $30 \mathrm{~min}$ per day with groups walking for more than $30 \mathrm{~min}$ a day. The group with low amount of walking time was more dependent as measured by the mRS and the Barthel Index and had a slower walking speed. These differences were not seen when comparing one group that walked for 30-60 min per day with another group who walked for more than $60 \mathrm{~min}$ per day. ${ }^{11}$ These results are in line with the findings in our study showing that being mostly active, as analysed in the first model, was important for staying active, whereas a higher PA level made no further contribution in predicting a higher PA level poststroke. Prestroke habits of PA may also possibly mean having some knowledge about PA and its beneficial health effects, whereas lack of knowledge and disbeliefs related to PA have been reported as barriers to PA by stroke survivors $^{2122}$ and could be a part of the explanation of our finding that prestroke PA level is important for being active after stroke.

The strength of this study was that many clinically important parameters that can be obtained early poststroke were considered as potential predictors for long-term outcome of PA level. It is of clinical importance to identify persons at risk of becoming inactive at an early stage, since PA after stroke may help in preventing secondary complications. ${ }^{4}$ Furthermore, the dichotomisations for PA level used in the study are clinically relevant and concurrent with recommendations for prevention of morbidity. There are, however, several limitations to this study, including a low number of cases in some subgroups that did not allow inclusion of all potential predictor variables into the regression models. The main outcome variable for PA was an interview based questionnaire. ${ }^{29} 30$ This type of scale presents with some problems including being at an ordinal level of data and the risk for recall bias. ${ }^{37}$ There is only a limited number of studies investigating validity of the six-graded scale used in this study. ${ }^{38}$ The dichotomisation used in the first model between grades 2 and 3 may, however, be directly translated into the original four-grade Saltin-Grimby Scale ${ }^{29}{ }^{30}$ which has been widely used and shown to have a good concurrent validity. ${ }^{38}$ Self-assessed PA has also been shown to have good predictor value for cardiovascular risk profiles ${ }^{39}$ as well as for functional outcome after stroke. ${ }^{19}$ The alternative option for reporting PA is direct measurement, for example, through using accelerometers. ${ }^{37}$ This option would not have been possible for establishing PA level prior to stroke, but could have been for outcome.

There are several other variables, such as mood, balance scales, ${ }^{40}$ fear of falling, ${ }^{20}$ lack of motivation and environmental factors ${ }^{21}$ that may influence PA after stroke that were not taken into account in the current study. Furthermore, our study based on the SALGOT cohort included only persons with an impaired upper extremity function 3 days poststroke, and the results apply only to those who were followed up at 1 year. Persons without impaired upper extremity might experience other obstacles for being physically active than people with upper limb impairment. Thus, the results from the current study can only be applied to persons showing at least some impairment of the upper extremity early after stroke and other studies are needed to see if the findings in our study may also apply to persons without upper extremity impairment early after stroke.

The present study aimed to identify persons that have a higher risk in becoming inactive after their stroke. The problem of inactivity among people with stroke is well established and recent recommendations have highlighted the challenges in increasing the PA among this group. ${ }^{4}$ By identifying which individuals who have an increased risk of becoming inactive after their stroke, allows clinicians to identify these persons earlier and so that targeted intervention could be offered as part of secondary prevention. ${ }^{4}$

\section{CONCLUSION}

Physical inactivity among stroke survivors is a major clinical problem. The present study indicates that persons with a higher age, higher degree of functional dependency early after stroke and a history of inactivity prior to stroke may have an increased risk of being insufficiently active at 1 year poststroke. These results may help to guide clinicians in identifying individuals in need of targeted interventions for reaching an adequate amount of PA; however, these findings need to be validated by other studies to show if the results may be applicable for other groups of stroke survivors. The list of predictor variables identified in this study contribute, but cannot explain all of the variation of PA level among stroke survivors and other predictors need to be further explored.

Contributors $\mathrm{OAO}, \mathrm{HCP}, \mathrm{MAM}$ and KSS contributed to the design of the study concept, in analysis and interpretation of results and in drafting/revising the manuscript for content. All authors have read and approved the final manuscript. In addition to this, HCP and MAM performed the acquisition of data. HCP, MAM and KSS obtained funding, OAO performed the statistical analysis and KSS supervised the SALGOT study.

Funding This study was funded in parts by the Swedish Research Council (VR 20123 70X32212230133), the Norrbacka-Eugenia Foundation, the Foundation of the Swedish National Stroke Association, Promobila, the Swedish Brain Foundation and the Swedish Heart-Lung Foundation.

Competing interests None declared.

Ethics approval The study was approved by the Regional Ethical Review Board in Gothenburg (225-08).

Provenance and peer review Not commissioned; externally peer reviewed.

Data sharing statement Interested researchers may submit requests for data to the authors (contact ks.sunnerhagen@neuro.gu.se). According to the Swedish regulation (http://www.epn.se/en/start/regulations/), the permission to use data is only for what has been applied for and then approved by the ethical board.

Open Access This is an Open Access article distributed in accordance with the Creative Commons Attribution Non Commercial (CC BY-NC 4.0) license, which permits others to distribute, remix, adapt, build upon this work non-commercially, and license their derivative works on different terms, provided the original work is properly cited and the use is non-commercial. See: http://creativecommons.org/ licenses/by-nc/4.0/

(C) Article author(s) (or their employer(s) unless otherwise stated in the text of the article) 2017. All rights reserved. No commercial use is permitted unless otherwise expressly granted. 


\section{REFERENCES}

1. O'Donnell MJ, Xavier D, Liu L, et al. Risk factors for ischaemic and intracerebral haemorrhagic stroke in 22 countries (the INTERSTROKE study): a case-control study. Lancet 2010;376:112-23.

2. Lee CD, Folsom AR, Blair SN. Physical activity and stroke risk: a meta-analysis. Stroke 2003;34:2475-81.

3. Wendel-Vos GC, Schuit AJ, Feskens EJ, et al. Physical activity and stroke. A meta-analysis of observational data. Int J Epidemiol 2004;33:787-98.

4. Billinger SA, Arena R, Bernhardt J, et al. Physical activity and exercise recommendations for stroke survivors: a statement for healthcare professionals from the American Heart Association/ American Stroke Association. Stroke 2014;45:2532-53.

5. World Health Organization. Global health risks: mortality and burden of disease attributable to selected major risks. Geneva 2009.

6. WHO. Global recommandations on physical activity for health. 2010 http://apps.who.int/iris/bitstream/10665/44399/1/9789241599979 eng.pdf (accessed 19 Aug 2015).

7. Krarup LH, Truelsen T, Gluud C, et al. Prestroke physical activity is associated with severity and long-term outcome from first-ever stroke. Neurology 2008;71:1313-8.

8. Deplanque D, Masse I, Libersa C, et al. Previous leisure-time physical activity dose dependently decreases ischemic stroke severity. Stroke Res Treat 2012;2012:1-6.

9. Hållmarker U, Åsberg S, Michaëlsson K, et al. Risk of recurrent stroke and death after first stroke in long-distance Ski race participants. $J$ Am Heart Assoc 2015;4:e002469.

10. Stroud N, Mazwi TM, Case LD, et al. Prestroke physical activity and early functional status after stroke. J Neurol Neurosurg Psychiatry 2009;80:1019-22.

11. Ursin $\mathrm{MH}$, Ihle-Hansen $\mathrm{H}$, Fure $\mathrm{B}$, et al. Effects of premorbid physical activity on stroke severity and post-stroke functioning. J Rehabil Med 2015;47:612-7

12. Bauman AE, Reis RS, Sallis JF, et al. Correlates of physical activity: why are some people physically active and others not? Lancet 2012;380:258-71.

13. Trost SG, Owen N, Bauman AE, et al. Correlates of adults' participation in physical activity: review and update. Med Sci Sports Exerc 2002;34:1996-2001.

14. Rand D, Eng JJ, Tang PF, et al. Daily physical activity and its contribution to the health-related quality of life of ambulatory individuals with chronic stroke. Health Qual Life Outcomes 2010;8:80.

15. English C, Manns PJ, Tucak C, et al. Physical activity and sedentary behaviors in people with stroke living in the community: a systematic review. Phys Ther 2014;94:185-96.

16. Danielsson A, Meirelles C, Willen C, et al. Physical activity in community-dwelling stroke survivors and a healthy population is not explained by motor function only. Pm $R$ 2014;6:139-45.

17. Danielsson A, Willén C, Sunnerhagen KS. Physical activity, ambulation, and motor impairment late after stroke. Stroke Res Treat 2012;2012:1-5.

18. Rand D, Eng JJ, Tang PF, et al. How active are people with stroke?: use of accelerometers to assess physical activity. Stroke 2009;40:163-8

19. López-Cancio E, Ricciardi AC, Sobrino T, et al. Reported prestroke physical activity is associated with vascular endothelial growth factor expression and good outcomes after stroke. J Stroke Cerebrovasc Dis 2017;26:425-30.
20. Roth EJ, Heinemann AW, Lovell LL, et al. Impairment and disability: their relation during stroke rehabilitation. Arch Phys Med Rehabil 1998;79:329-35.

21. Nicholson S, Sniehotta FF, van Wijck F, et al. A systematic review of perceived barriers and motivators to physical activity after stroke. Int $J$ Stroke 2013;8:357-64.

22. Jackson S, Mercer C, Singer BJ. An exploration of factors influencing physical activity levels amongst a cohort of people living in the community after stroke in the south of England. Disabil Rehabil 2016:1-11.

23. Alt Murphy M, Persson HC, Danielsson A, et al. SALGOT--Stroke arm longitudinal study at the University of Gothenburg, prospective cohort study protocol. BMC Neurol 2011;11:56.

24. Lyle RC. A performance test for assessment of upper limb function in physical rehabilitation treatment and research. Int J Rehabil Res 1981;4:483-92.

25. von Elm E, Altman DG, Egger M, et al. The strengthening the reporting of observational studies in epidemiology (STROBE) statement: guidelines for reporting observational studies. Lancet 2007;370:1453-7.

26. Asplund K, Hulter Åsberg K, Appelros P, et al. The Riks-Stroke story: building a sustainable national register for quality assessment of stroke care. Int J Stroke 2011;6:99-108.

27. Brott T, Adams HP, Olinger CP, et al. Measurements of acute cerebral infarction: a clinical examination scale. Stroke 1989;20:864-70.

28. van Swieten JC, Koudstaal PJ, Visser MC, et al. Interobserver agreement for the assessment of handicap in stroke patients. Stroke 1988;19:604-7.

29. Grimby G. Physical activity and muscle training in the elderly. Acta Med Scand Suppl 1986;711:233-7.

30. Mattiasson-Nilo I, Sonn U, Johannesson K, et al. Domestic activities and walking in the elderly: evaluation from a 30-hour heart rate recording. Aging 1990;2:191-8

31. Ladenvall P, Persson CU, Mandalenakis Z, et al. Low aerobic capacity in middle-aged men associated with increased mortality rates during 45 years of follow-up. Eur J Prev Cardiol 2016;23:1557-64.

32. Takagi D, Nishida Y, Fujita D. Age-associated changes in the level of physical activity in elderly adults. J Phys Ther Sci 2015;27:3685-7.

33. Kunkel D, Fitton C, Burnett M, et al. Physical inactivity post-stroke: a 3-year longitudinal study. Disabil Rehabil 2015;37:304-10.

34. Lindahl M, Hansen L, Pedersen A, et al. Self-reported physical activity after ischemic stroke correlates with physical capacity. Adv Physiother 2008;10:188-94.

35. Buchman AS, Wilson RS, Yu L, et al. Total daily activity declines more rapidly with increasing age in older adults. Arch Gerontol Geriatr 2014;58:74-9.

36. Knoflach M, Matosevic B, Rücker M, et al. Functional recovery after ischemic stroke--a matter of age: data from the Austrian Stroke Unit Registry. Neurology 2012;78:279-85.

37. Hagströmer M, Oja P, Sjöström M. Physical activity and inactivity in an adult population assessed by accelerometry. Med Sci Sports Exerc 2007;39:1502-8.

38. Grimby G, Börjesson M, Jonsdottir IH, et al. The "Saltin-Grimby Physical Activity Level Scale" and its application to health research. Scand J Med Sci Sports 2015;25(Suppl 4):119-25.

39. Thune I, Njølstad I, Løchen ML, et al. Physical activity improves the metabolic risk profiles in men and women: the Troms $\varnothing$ Study. Arch Intern Med 1998;158:1633-40.

40. Alzahrani MA, Dean CM, Ada L, et al. Mood and balance are associated with free-living physical activity of people after stroke residing in the community. Stroke Res Treat 2012;2012:1-8. 